\title{
33 Die Krim im Zweiten Weltkrieg
}

\author{
Von unten näselt das Akkordeon ,Die Wacht am Rhein‘. \\ Dunkelheit. Schatten eines deutschen Bajonetts. \\ Um Mitternacht weckt der alte Matrose der Schwarzmeerflotte Koška \\ den Matrosen der Roten Flotte Ševčuk. \\ Und sie gehen von Inkerman, \\ durch finstere Totenstille \\ auf den Malachov-Hügel \\ vorbei am Korabel'noj-Viertel ${ }^{1}$
}

Der vor allem als Verfasser patriotischer Reime und Kriegskorrespondent im Zweiten Weltkrieg berühmt gewordene sowjetische Schriftsteller Aleksej A. Surkov (1899-1983) war in der UdSSR einer der höchstausgezeichneten Literaten. In der Nachfolge des in der Sowjetunion so verehrten Maksim Gorki (1868-1936) stand er von 1953 bis 1959 sogar dem Schriftstellerverband der UdSSR vor. In dem Gedicht „Die Sevastopol'er“ („Sevastopol'cy“) von 1943, dessen erste zwei Strophen hier zitiert werden, fließen zwei Zeitebenen zusammen - für die sowjetische Leserschaft der Zeit gut verständlich -, nämlich die des Krimkriegs von 1853 bis 1856 mit der des Zweiten Weltkriegs. Bei Surkov ,weckt' einer der bekanntesten Helden des Krimkriegs, der Matrose Pëtr M. Koška (1828-1882), einen Matrosen der Roten Flotte, um mit ihm „durch finstere Totenstille“ an den Feinden vorbei auf den Malachov-Hügel, einen der blutigsten Schauplätze des Krimkrieges, zu gehen und schließlich in einem Bezirk Sevastopol's mit dem Namen Korabel'noj zu landen; von ferne hört man das Lied „Die Wacht am Rhein“, welches im Deutschen Kaiserreich als eine Art inoffizielle Nationalhymne und im Ausland als Signum deutschen Eroberungswillens galt. Der Zweite Weltkrieg begann für die Sowjetunion insgesamt und auch für die BewohnerInnen der Krim im Juni 1941 mit ersten Angriffen der deutschen Luftwaffe auf Sevastopol'. Ein militärisch und erinnerungskulturell wirkungsmächtiger Höhepunkt des Kampfes um die Halbinsel wurde die 250 Tage währende Belagerung Sevastopol's durch die Wehrmacht, die vom 30. Oktober des Jahres 1941 bis Anfang Juli 1942 dauerte. Erst dann konnten deutsche Truppen Vollzug nach Berlin vermelden, nämlich die Besetzung der ganzen Halbinsel, bevor Anfang 1944 mit dem Rückzug begonnen werden musste. Die ,Heldenstadt' Sevastopol' hielt im 20. Jahrhundert zwar knapp hundert Tage kürzer der Belagerung der Gegner stand als im Krimkrieg, gleichwohl wurde der mindestens ebenso tapfere Opferwille des Sowjetvolks -

1 Surkov (1989), 137.

Ә OpenAccess. (C) 2020 Kerstin S. Jobst, publiziert von De Gruyter. (cc))BY Dieses Werk ist lizenziert unter der Creative Commons Attribution 4.0 International. https://doi.org/10.1515/9783110520620-035 
zumal in der konstruierten Gleichzeitigkeit im Gedicht Surkovs - in heroischer Diktion beschworen.

Die Heldennarration Sevastopol's hat den Systembruch vom Zaren- zum Sowjetreich und letztlich bis in unsere Zeit unbeschadet überstanden. Auf einer russischen Website mit dem Titel „Wargaming.net“ heißt es unter dem Eintrag zu Pëtr M. Koška beispielsweise, dass nur diejenigen diesen Matrosen nicht kennen, die niemals von der „,heldenhafte Verteidigung“ der Stadt in den Jahren 1854-1855 gehört hätten, was aber - so die Vermutung des unbekannten Verfassers - eigentlich unmöglich sei. ${ }^{2}$ Um noch einmal auf Surkovs Gedicht zurückzukommen: Es ist bemerkenswert, dass der sowjetische Autor beileibe nicht nur den einfachen Matrosen Koška erwähnte und damit die im Mittelpunkt der Ideologie stehenden Werktätigen, sondern auch sozial und militärisch hochgestellte, aber verdiente Helden der Schwarzmeerflotte: Den Weltumsegler, Forscher und Kommandeur der Schwarzmeerflotte Michail P. Lazarev (1788-1851) beispielsweise oder die im Krimkrieg gefallenen Offiziere Vladimir A. Kornilov (1806-1854, Vize-Admiral) und Vladimir I. Istomin (1809-1855, Konter-Admiral), die beide auf dem Malachov-Hügel tödliche Verwundungen davontrugen. Die Zusammenführung unterschiedlicher Zeitebenen (hier also des Krimkrieges mit dem Zweiten Weltkrieg) hilft, eine lineare Geschichtserzählung zu konstruieren. Dies ist ein Stilmittel der Geschichtspolitik sowohl der Sowjetunion als auch der Russländischen Föderation, spielt aber auch in anderen nationalen Kontexten eine Rolle. Signifikant ist überdies - darauf hat Serhii Plokhy schon Jahre vor der Annexion der Krim 2014 hingewiesen -, dass Sevastopol' in diesen Erzählungen zu einer exklusiv russischen Stadt wird; sogar der Anteil der ukrainischstämmigen Bevölkerung wird unterschlagen. ${ }^{3}$

Das deutsche Besatzungsregime brachte die ganze Bandbreite des nationalsozialistischen Vernichtungskriegs gegen die Sowjetunion auch auf die Krim - Gewalt, Judenvernichtung, Zwangsarbeit, Kollaboration, Ausbeutung, Passivität und Partisanentätigkeit prägten die Verhältnisse. Die ersten Opfer im Krieg waren die Krimdeutschen. Diese hatten nach den Zahlen der ersten Volkzählung im Zarenreich von 1897 5,78 Prozent der Bevölkerung im Taurischen Gouvernement gestellt, welches aber, wie erwähnt, größer als die Halbinsel war, ${ }^{4}$ so dass eine exakte Schätzung schwerfällt. Bei Kriegsbeginn lebten vermutlich mehr als 53.000 Deutsche auf der Krim. Im August 1941, also noch bevor die Wehrmacht die

2 Koška (2017).

3 Plokhy (2000), 372.

4 Pervaja perepis' (2018). 
Halbinsel erreicht hatte, verfügte Stalin deren Umsiedlung in den frontfernen Osten der Sowjetunion, um die - wie bereits im Ersten Weltkrieg (Kapitel 31) gefürchtete Kollaboration mit den deutschen Angreifern zu verhindern. Viele von ihnen landeten in der sog. Trudarmija (russ.; eig. Trudovaja armija; Arbeitsarmee), einer Anfang der 1920er Jahre erstmalig eingeführten und im Zweiten Weltkrieg neu belebten Form der Zwangsarbeit. ${ }^{5}$

Im Herbst 1941 (zum Zeitpunkt des deutschen Einmarsches) befanden sich nur knapp 1.000 sog. Krimdeutsche auf der Halbinsel, so dass sie allein aufgrund ihrer geringen Zahl nur bedingt als Nukleus für die von den Nationalsozialisten geplante Germanisierung in Betracht kamen. Dieser Umstand musste die tollkühnen Pläne der Nationalsozialisten zur Errichtung des sog. Gotengaus (Kapitel 2) also beeinflussen.

Im Vernichtungskrieg gegen die Sowjetunion hatte die Halbinsel trotz wolkiger Träumereien von einer ,deutschen Krim‘ anfänglich keine zentrale Rolle gespielt, da ihr militärisch ein „Sackgassencharakter“ zugesprochen worden war. ${ }^{6}$ Durch den bald gestoppten Vormarsch Richtung Osten wurde dies aber alsbald von den deutschen Strategen als Vorteil gedeutet. Auch als Ausgangspunkt der geplanten Einnahme kriegswichtiger Ölfelder im nördlichen und südlichen Kaukasus wurde der Krim Bedeutung zugemessen. Zudem hofften Berliner Stellen, mit ihrem Besitz einen Unterpfand in den Händen zu halten, durch den sich die offiziell neutrale Türkei dem Deutschen Reich annähern würde. Dass für diese „die Krim dagegen kein Interessengebiet erster Wahl“ war, ${ }^{7}$ enttäuschte nicht nur die Deutschen, sondern auch exilierte KrimtatarInnen wie Seydahmet, die in Polen $^{8}$, Rumänien, aber auch in Istanbul, Ankara und sogar im Libanon dezidiert antisowjetische Ziele verfolgten. ${ }^{9}$ Vergeblich hofften sie auf die aktive Unterstützung Ankaras für die Sache der KrimtatarInnen. Nationale Akteure wie der in der Dobrudscha geborene Müstecib Ülküsal (1899-1996) oder der nach der Hinrichtung İbraimovs und seiner Mitstreiter (Kapitel 32) von der Halbinsel geflohene Mustafa Edige Q1rımal (= Edige Kirimal; 1911-1980) bemühten sich nach dem Sommer 1941 in Berlin sogar um die Erlaubnis zur Organisierung der krimtatarischen Bevölkerung und um die Freilassung krimtatarischer Rotarmisten aus

5 Dazu u.a. Eisfeld u. Herdt (1996). Vgl. auch den Bericht einer betroffenen Krimdeutschen: Riss (2007/2008).

$6 \operatorname{Kunz}(2005), 16$.

7 Kunz (2005), 27.

8 Krimtatarische Exilanten wirkten auch in der von Polen unterstützten antisowjetischen Prometheus-Bewegung mit, Copeaux. Vgl. auch Gasimov (2011).

9 Vgl. zu den transnationalen Netzwerken der krimtatarischen Diaspora in der Zwischenkriegszeit Gasimov (2017). 
deutscher Kriegsgefangenschaft. Es sollte sich aber wie im Ersten Weltkrieg (Kapitel 31) zeigen, dass die deutsche Seite auf die Angebote zur Kollaboration nur dann einging, wenn es ihren Interessen nützte. Einen unabhängigen krimtatarischen Staat unterstützen die Deutschen keinesfalls. ${ }^{10}$ Der sowjetischen Regierung boten solche Aktionen allerdings Anlass, ${ }^{11}$ nach dem Krieg die Unzuverlässigkeit der ganzen krimtatarischen Nationalität zu behaupten, was im Kontext der Deportationen 1944 (Kapitel 34) noch auszuführen ist. In jedem Fall ging es weder Hitler noch den zahlreichen anderen nachgeordneten Stellen bei der Besetzung der Krim um die Befreiung der KrimtatarInnen.

Nicht zu unterschätzen war der Nimbus Sevastopol's, welches sowohl von den Sowjets als auch deutscherseits als eine der am besten befestigten Militäranlage der Welt bezeichnet wurde; für die Angreifer stand also, wie Norbert Kunz auf den Punkt bringt, „bei der Eroberung der Stadt einiges Prestige auf dem Spiel.“12 Tatsächlich machten es die Frauen und Männer Sevastapol's den Angreifern nicht leicht, widerstanden sie doch 250 Tage, was den deutschen Triumph dann umso größer erscheinen ließ.

Der Marsch auf die Krim hatte für die Wehrmacht nach der erfolgreichen Schlacht am Azovschen Meer im Herbst 1941 begonnen. Die unter dem Befehl des neuinstallierten Generals Erich von Manstein (1887-1973) stehende 11. Armee ${ }^{13}$ stieß an der Seite der verbündeten 3. Rumänischen Armee über die Landenge von Perekop auf die Halbinsel vor. Ende Oktober begann die Belagerung Sevastopol's, an der sich rumänische Streitkräfte abermals beteiligten. Auch im Osten, auf der Halbinsel Kerč', setzte sich die Wehrmacht anfänglich nicht durch, war doch zum Jahreswechsel 1941/42 die Rote Armee zurückgekehrt. Das sog. Unternehmen Trappenjagd im Mai 1942 endete für die deutsch-rumänischen Verbände schließlich mit der Einnahme von Kerč’ ${ }^{14} 100.000$ Rotarmisten gerieten in Gefangenschaft. Das „Unternehmen Störfang“ schließlich, dessen Verlauf der Mittelalterhistoriker Percy Ernst Schramm (1894-1970) als Kriegstagebuchschreiber im Oberkommando der Wehrmacht aus der Sicht der obersten Führung festgehalten hat, ${ }^{15}$ führte Anfang Juli 1942 zum Fall Sevastopol's. Doch der Preis war

10 Vgl. zu dieser Berlin-Reise Aydin (2002); Williams (2001), $377 \mathrm{f}$.

11 Kirimal beispielsweise, der nach dem Krieg in der Bundesrepublik Deutschland verblieb, war u. a. Mitglied der Waffen-SS. Vgl. Roman'ko (2004). Vgl. zudem Roman'ko (2011).

12 Kunz (2005), 18.

13 Der vorherige Befehlshaber Eugen Siegfried Erich Ritter von Schobert (1883-1941) war kurz vorher bei einem Unfall ums Leben gekommen.

14 Vgl. z. B. die apologetische Deutung Erich von Mansteins: Manstein (2004), 256-261.

15 Schramm (1982). Zur Bewertung vgl. Messerschmidt (2004). 
hoch: Manstein und die rumänischen und nun auch italienischen Angreifer ${ }^{16}$ opferten über ein Drittel ihrer Angriffsstärke.

Auch auf der Krim wurde schon beim deutschen Vormarsch die tödliche Bedrohung der lokalen jüdischen Bevölkerung sichtbar: Auch dort wurden sog. Einsatzgruppen (EG) - im Falle der südlichen Sowjetunion war dies die EG D unter Otto Ohlendorf (1907-1951) - als Vor- oder auch Nachhut der Armee eingesetzt. Neben der Ausschaltung angeblicher oder tatsächlicher Partisanen und kommunistischer Funktionsträger gehörte die Ermordung von Juden und Roma zu ihren ,Aufgaben'. Im sog. Massaker von Simferopol' im Dezember 1941 hatte die Einsatzgruppe D bereits schreckliche Berühmtheit erlangt. Unmittelbar nach der Eroberung Sevastopol's wurden über viertausend Jüdinnen und Juden massakriert. ${ }^{17}$ Dies war allerdings erst das Vorspiel zur systematischen rassistischen Ausrottungspolitik.

Für Manstein war der Krim-Feldzug ein hart errungener Erfolg, für den er mit dem Rang eines Generalfeldmarschalls belohnt wurde. Und für die überlebenden Wehrmachtssoldaten kreierte die Wehrmachtsführung eine eigene Auszeichnung, den Krimschild. Propagandistisch wurde die Einnahme der vermeintlich uneinnehmbaren Feste Sevastopol' weidlich ausgeschlachtet: Pressevertreter und Diplomaten aus dem Reich und den verbündeten Ländern besuchten die zerstörte Stadt, und selbst der rumänische König Michael I. (1921-2017) kam. ${ }^{18}$

In seiner verdienstvollen Studie über die Jahre 1941 bis 1944 auf der Krim kommt Kunze zu dem Ergebnis, dass unter Manstein ein „in mancher Hinsicht moderateres Besatzungsregime“ herrschte als in vielen anderen Teilen der von den Deutschen überrannten Gebieten der Sowjetunion. ${ }^{19}$ Dieser Aussage kann in Anbetracht der verbrecherischen Politik des Dritten Reiches insgesamt nicht zugestimmt werden. Dies zumal, wenn man der recht erfolgreichen apologetischen Selbststilisierung des Generalfeldmarschalls Manstein nach dem Krieg nicht folgen mag, dem nach seiner Entlassung aus der Kriegsgefangenschaft Anfang der 1950er Jahre übrigens eine zweite Karriere als inoffizieller Berater beim Aufbau

16 In dem militärgeschichtlich und national-apologetisch ausgerichteten Werk des russischen Autors A.B. Širokorad (2006), 233-248, widmet sich dieser dem italienischen Anteil des Krimfeldzuges, indem er vor allen Dingen das eingesetzte Material auflistet. Interessant ist die Überschrift dieses Kapitels: „Die ungewöhnlichen Abenteuer der Italiener in Russland“ („Neobyknovennye priključenija ital'jancev $v$ Rossii“), erinnert er doch damit an eine in der Sowjetunion sehr populäre Komödie aus dem Jahr 1974 - „Die ungewöhnlichen Abenteuer von fünf Italienern in Russland“.

17 Vgl. Angrick (2003), besonders 324-360.

18 Kunz (2005), $18 \mathrm{f}$.

19 Kunz (2005), 236. 
der Bundeswehr gelang. Er verstand es, einerseits sich der bundesrepublikanischen Öffentlichkeit gegenüber als militärischer Experte zu präsentieren und andererseits das Bild eines von den Niederungen der Politik unbeleckten, ,sauberen' Wehrmachtssoldaten zu stilisieren - im Gegensatz zur ,verbrecherischen SS oder den Einsatzgruppen. ${ }^{20}$ Beides waren Interpretationsangebote, denen die Bevölkerung der Bundesrepublik Deutschland der Nachkriegszeit mehrheitlich nur zu gern folgte.

Nach dem Überfall auf die Sowjetunion im Juni 1941 war die Wehrmacht in manchen Gebieten (z.B. in dem seit Ende 1939 unter sowjetischer Verwaltung stehenden ehemaligen Ostgalizien) in Verkennung der eigentlichen Ziele des nationalsozialistischen Staates freundlich empfangen worden. Auch auf der Halbinsel begrüßten Teile der verbliebenen, $d . h$. nicht in der sowjetischen Armee dienenden Krimtataren bzw. die wenigen nicht deportierten sog. Volksdeutschen die Wehrmacht in positiver Erwartung. Die seit Mitte der 1930er Jahre erlittene Verfolgung, der auch Vertreter der vordem geförderten krimtatarischen Kultur wie der Turkologe und Schriftsteller Bekir Çoban-zade (1893-1937) zum Opfer gefallen waren, ${ }^{21}$ mag dies erklären. Hinzu kamen die - zumindest in den ersten Monaten der Besatzung - pragmatisch motivierten Zugeständnisse der deutschen Politik. Insgesamt ist dem Urteil Johannes Hürters über die nationalsozialistische Politik gegenüber den nichtrussischen Nationalitäten im Besatzungsgebiet zuzustimmen: Diese sei ein nur „halbherziges Werben um die einheimische Bevölkerung“ gewesen, der unzureichende Angebote hinsichtlich der Agrarpolitik, Selbstverwaltung und Religion gemacht wurden, die deshalb „ins Leere laufen“ mussten.22 Die nationalsozialistische Politik war wesentlich von rassistischen Urteilen und Leitlinien geprägt, die weitergehende Angebote an die Nationalitäten verhinderten, die als minderwertig angesehen wurden. Die nationalsozialistische KrimPolitik war jedoch nicht allein rasseideologisch oder strategisch motiviert: Auch die Berlin nicht verborgen gebliebene Schönheit, ihre nun kriegswirtschaftlich wichtig eingeschätzte Lage im Rahmen des „Generalplans Ost“ des Reichssicherheitshauptamtes (RSHA) sowie die Gotengau-Phantasien Hitlers beeinflussten diese. Die partiell

weniger restriktive Politik gegenüber der krimtatarischen Bevölkerung (etwa durch die partielle Förderung tatarischer kultureller Aktivitäten oder durch Zugeständnisse in der Selbstverwaltung) ist als Teil eines in Besatzungsregimen notwendigen Kooperationsangebotes $\mathrm{zu}$ verorten, nicht als positive Tatarenpolitik. Letztlich war KrimtatarInnen in den

20 Dazu ausführlich Wrochem (2006).

21 Vgl. Bowman (2003).

22 Hürter (2012), 25. 
nationalsozialistischen Umsiedlungsplänen mittelfristig nämlich ein ähnliches Schicksal zugedacht wie anderen ,nichtarischen' Gruppen. ${ }^{23}$

Dies hieß letztlich Deportation, auch wenn laut dem Anfang Juli 1942, also noch vor der Einnahme Sevastopol's, von Hitler erlassenen Deportationsbefehl nur „alle Russen, Armenier und sonstige Bolschewisten“ umgesiedelt werden sollten, nicht aber KrimtatarInnen oder Krimdeutsche; in einem ersten Entwurf war allerdings von Krim-Muslimen die Rede gewesen. ${ }^{24}$ Der „Führer“ konnte von den Akteuren vor Ort nur mit Mühe von diesen Plänen abgebracht werden, da die Umsiedlung von ca. drei Viertel der gesamten Krim-Bevölkerung logistisch und zumal unter den Bedingungen des Krieges schwer zu bewerkstelligen gewesen wäre. Überdies wäre diese Entvölkerung für die Ziele des Reiches kontraproduktiv gewesen, u.a. da der Halbinsel im nationalsozialistischen Raub- und Ernährungskrieg eine hervorragende Rolle zugesprochen wurde. Der Verlust eines großen Teils der Erntearbeitskräfte hätte nicht ausgeglichen werden können und in der Tat wurde der Befehl aus Nützlichkeitserwägungen zurückgezogen. ${ }^{25}$

Mehr Pragmatismus in der letztlich auf Entrechtung, wenn nicht gar Ausrottung abzielenden Bevölkerungspolitik wünschten nicht nur die militärischen Akteure vor Ort. Ähnlich dachte auch der Gesandte Werner Otto von Hentig (18861984), der dem Auswärtigen Amt im April 1942 die negativen Folgen des Judenmords auf der Krim schilderte: Er berichtete nach Berlin von den Massenerschießungen an Jüdinnen und Juden - allein in Simferopol' 12.000 in kürzester Zeit - und beklagte die „Wirkungen einer solchen Schlächterei.“ Diese beschränkten

sich ja keineswegs auf die Opfer selbst; sie berühren einmal die gesamte Bevölkerung des besetzten Gebietes, weil es natürlich keiner für möglich gehalten hat, daß wir Frauen und Kinder töten. Sie berühren aber auch die Moral der Truppen und weiterhin auch unsere wirtschaftliche Stellung. Von den Wirkungen im weiteren Ausland natürlich vollkommen abgesehen.

Wirtschaftlich sei das Vorgehen ebenfalls negativ zu bewerten, da in den Städten der Region Juden häufig im Handwerk tätig gewesen seien, deren Fähigkeiten und Arbeitskraft nun fehlten. ${ }^{26}$ Solche raren Stimmen blieben ungehört, denn auch auf der Krim wurde die Shoah rücksichtslos exekutiert, so dass bereits nach nur sechs Monaten nach Berlin gemeldet werden konnte, die Halbinsel sei nun ,ju-

23 Jobst (2017a), 106.

24 Zitiert nach Kunz (2005), 65.

25 Kunz (2005), 66.

26 Hentig (2018). 
denfrei“. Dem vorangegangen waren die ,üblichen` Entrechtungen, Stigmatisierungen und Demütigungen der jüdischen Bevölkerung sowie die Verbrämung des Ausrottungswillens der Besatzungsmacht, welche die bevorstehende Ermordung als „Umsiedlung“ bezeichnete. Auch auf der Krim unterstützte die Wehrmacht den Völkermord der Einsatzgruppen. Unterschiede zu anderen Schauplätzen des nationalsozialistischen Genozids existierten indes ebenfalls ${ }^{27}$ : Aufgrund der Geographie der Halbinsel wurden die lokalen Jüdinnen und Juden nicht erst in Konzentrationslager gebracht, sondern vor Ort ermordet und verscharrt. Wohl auch, um die psychischen Belastungen der Täter zu verringern - die der Opfer spielte in der nationalsozialistischen Vernichtungslogik kaum überraschend keine Rolle -, wurde auf der Krim ausführlich mit Tötungsmaschinen experimentiert, die von Einheimischen „dušegubka“ (russ.; „Seelentöter“) genannt wurden. Neben Gaswagen sind u. a. Kähne zu nennen, die mitsamt den darauf befindlichen Menschen im Schwarzen Meer versenkt wurden; mehr als tausend Menschen kamen so zu Tode. Anders als beispielsweise im ehemaligen Ostgalizien $^{28}$ kam es auf der Krim aber nicht zu lokalen Pogromen gegen die jüdischen Nachbarn, was allerdings vereinzelte antisemitische Taten tatarischer, aber auch slavischer KrimbewohnerInnen nicht ausschloss. ${ }^{29}$

Der wohl wesentliche Unterschied zur Shoah im übrigen Okkupationsgebiet war das sich den nationalsozialistischen Akteuren vor Ort und den Rassekundlern im fernen Berlin präsentierende „Durcheinander“, wie Kunz es lax, aber treffend ausgedrückt hat. ${ }^{30}$ Durch die von den Nationalsozialisten beanspruchte Definitionsmacht konnten sie entscheiden, wer als Jude zu gelten hatte oder nicht. Die eindimensionale Reduktion auf das obskure Merkmal ,Rasse' wurde der Vielfältigkeit des Judentums nicht gerecht. Während die Unterschiede zwischen Aschkenasim und Sephardim den Nationalsozialisten bekannt waren und Angehörige beider Gruppen gleichermaßen der Shoah zum Opfer fielen, stellte der Formenreichtum jüdischen Lebens auf der Krim diese vor ein Definitionsdilemma. Neben den talmudistischen Jüdinnen und Juden (ca. 60.000), die auf der Krim überwiegend Aschkenasim waren und umstandslos der Vernichtung anheimgefallen sind, existierten noch drei weitere Gruppen: Bergjuden, sog. Krimčaken (ca. 6.400) sowie die bereits erwähnten Karäer (Kapitel 25), die mit ca. 8.300 Personen den größten dieser kleineren Zweige repräsentierten. ${ }^{31}$

27 Ich folge im Wesentlichen Kunz (2005), 179-204.

28 Vgl. dazu Struve (2015).

29 Dazu aber auch zur antisemitischen Propaganda der Besatzungsmacht auf der Krim vgl. Tyaglyy (2011).

30 Kunz (2005), 191.

31 Ich folge hier Green (1984), 171. Alle Zahlen beziehen sich auf die Zeit vor 1941. 
Die ursprünglich aus dem Kaukasus stammenden Bergjuden waren erst Ende der 1930er Jahre durch die Unterstützung des zwischen 1924 und 1938 auch in der Sowjetunion tätigen American Jewish Joint Distribution Committee auf die Krim gelangt, und zwar im Rahmen der sog. Produktivierungskampagne. An deren Jüdischsein' hegten die verantwortlichen deutschen Täter keinen Zweifel, und somit wurden sie Opfer der Shoa. ${ }^{32}$ Anders sah die Lage hinsichtlich der Krimčaken, einer sprachlich tatarisierten jüdischen Gruppe, und der Karäer aus. Ohlendorf selbst nahm diese beiden im Dezember 1941 vorerst von der Vernichtung aus und wandte sich hilfesuchend an den Chef des für „Rassefragen“ zuständigen „Reichsministeriums für die besetzten Ostgebiete“, Alfred Rosenberg, mit der Bitte, deren „Rassenzugehörigkeit“ zu prüfen. In Berlin kam man zu dem Ergebnis, dass erstere in jedem Fall Juden seien. Mit ihrer Vernichtung wurde sogleich begonnen, und wie auch in anderen Teilen des besetzten Europas konnten sich nur wenige von ihnen durch Verstecken retten. ${ }^{33}$ Im Fall der Karäer kam man hingegen zu einem anderen Urteil, denn wie bereits die zarischen Behörden so gingen auch die Deutschen letztlich davon aus, dass es sich bei den Karäern nicht um Juden handele. Wie Kiril Feferman ausführt, befassten sich deutsche Rassepolitiker und Wissenschaftler bereits in den 1930er Jahr mit der Karäer-Frage - und kamen dabei zu divergierenden Ergebnissen. Es war ein Glück für die Krim-Karäer, dass während der Besatzung der Halbinsel die Auffassung überwog, diese seien keine Juden, wobei die Deutschen sich auf ,wissenschaftliche' Urteile als auch auf Selbstauskünfte aus den Reihen dieser Gruppe verließen. ${ }^{34}$ Immer wieder wird in der Literatur darauf verwiesen, dass Mitglieder der anderen jüdischen Gemeinden sich für ihre Glaubensverwandten eingesetzt hätten - indem sie bestätigten, bei den Karäern handele es sich eben nicht um Juden. ${ }^{35}$ Wie auch immer, der „Reichsführer SS“ selbst, Heinrich Himmler (19001945), entschied schließlich zu ihren Gunsten, was für Feferman beweist, welche Bedeutung der NS-Staat der Karäer-Frage zuwies. ${ }^{36}$ Dass dennoch vereinzelt Karäer auf der Krim Opfer der Nationalsozialisten wurden, war zum Teil schlicht ein Versehen. In jedem Fall scheinen bei der Verschonung der Krim-Karäer pragmatische Erwägungen die deutsche Politik bestimmt zu haben, da sie „den Rückhalt insbesondere der tatarischen Nachbarn genossen, eines aus deutscher Sicht unverzichtbaren Partners also.“37 Möglicherweise wäre es, wenn es denn zum sog.

32 Feferman (2007), besonders 104.

33 Vgl. Green (1984), $172 f$.

34 Feferman (2011), $283 \mathrm{f}$.

35 Vgl. z. B. Ascherson (1996), 45.

36 Feferman (2011), 284.

37 Kunz (2005), 190. 
Endsieg für das Deutsche Reich gekommen wäre und keine Rücksichten mehr genommen werden müssten, zur Neubewertung der ,Rassenzugehörigkeit' der Karäer gekommen. Vorerst waren diese aber nützlich, zumal einige von ihnen in deutsche Dienste traten und im April 1944, als die Wehrmacht den Rückzug antrat, mit dieser die Krim in Richtung Westen verließen. ${ }^{38}$

Über die Unterstützung der Besatzungsmacht durch die lokale Bevölkerung wird im Kontext der Deportationen nach der Rückeroberung der Halbinsel durch die sowjetische Armee zu sprechen sein (Kapitel 34). Hier sei schon erwähnt, dass die Begeisterung vieler Kollaborateure der ersten Stunde schnell in Enttäuschung umschlug. Auch die nicht per se aufgrund dubioser Zuordnungen von Wehrmacht und Einsatzgruppen verfolgten Gruppen spürten bald die Brutalität des Regimes. Razzien, Kontrollen und Nahrungsmittelengpässe prägten den Alltag. Da die fruchtbare Krim für die Versorgung der Soldaten an der Ostfront eine große Rolle spielte, wurden viele Lebensmittel abgezogen. ${ }^{39}$ Bis zum Rückzug der Wehrmacht stand die Krim wegen der verschärften Sicherheitslage unter strenger militärischer Verwaltung: Auf der Krim und zwar besonders im für die herkömmliche Kriegführung schlecht zugänglichen Gebirge, tobte ein erbitterter Partisanenbzw., wie es in der Sprache der Nationalsozialisten hieß, „Bandenkrieg“. Auch wenn die Besatzer phasenweise den Widerstandskämpfern erheblich zusetzten, letztlich waren die deutschen Kräfte diesen und vor allen Dingen den sowjetischen Truppen nicht gewachsen. Im April 1944 musste der Rückzug angetreten werden. Und die Krim wurde wieder sowjetisch. ${ }^{40}$

38 Feferman (2011), 285, schreibt von „hundreds of Karaites.“

39 Kunz (2005), besonders 133-154.

40 Kunz (2005), besonders 109-132. 